\title{
Manipulating the Magnetic Structure with Electric Fields in Multiferroic $\operatorname{ErMn}_{2} \mathrm{O}_{5}$
}

\author{
Y. Bodenthin, ${ }^{1}$ U. Staub, ${ }^{1}$ M. García-Fernández, ${ }^{1}$ M. Janoschek,,${ }^{2,3}$ J. Schlappa, ${ }^{1}$ E. I. Golovenchits, ${ }^{4}$ \\ V. A. Sanina ${ }^{4}$ and S. G. Lushnikov ${ }^{4}$ \\ ${ }^{1}$ Swiss Light Source, Paul Scherrer Institut, CH-5232 Villigen PSI, Switzerland \\ ${ }^{2}$ Laboratory for Neutron Scattering, ETH Zurich and Paul Scherrer Institut, CH-5232 PSI, Villigen, Switzerland \\ ${ }^{3}$ Physik Department E21, TU München, 85748 Garching, Germany \\ ${ }^{4}$ Ioffe Physical Technical Institute, 26 Politekhnicheskaya, 194021 St. Petersburg, Russia
}

(Received 11 July 2007; published 15 January 2008)

\begin{abstract}
Based on measurements of soft X-ray magnetic diffraction under in situ applied electric field, we report on significant manipulation and exciting of commensurate magnetic order in multiferroic $\mathrm{ErMn}_{2} \mathrm{O}_{5}$. The induced magnetic scattering intensity arises at the commensurate magnetic Bragg position whereas the initial magnetic signal almost persists. We demonstrate the possibility to imprint a magnetic response function in $\mathrm{ErMn}_{2} \mathrm{O}_{5}$ by applying an electric field.
\end{abstract}

DOI: 10.1103/PhysRevLett.100.027201

The coexistence of magnetism and ferroelectricity in solid materials is unusual, and still more unusual is the coupling between these phenomena. Materials with such couplings are often called multiferroics, and the coupling is based on the magneto-electric effect. The interest in these materials lies in the fact that a manipulation of electric (magnetic) properties by magnetic (electric) fields would be of great use in spintronic devices [1-3]. In recent years, it has been shown that noncollinear, long-range antiferromagnetic structures can break spatial inversion symmetry and drive a ferroelectric modification [4,5]. Ferroelectricity may arise regardless of the commensurability between the lattice and magnetic order [6]. Electric polarization produced by the spin current (vector spin chirality) $\mathbf{S}_{\mathbf{i}} \times$ $\mathbf{S}_{\mathbf{j}}$ may even arise in the presence of a center of inversion symmetry between spins, i.e., without DzyaloshinskiiMoriya interaction, with a noncollinear configuration such as the transverse-spiral and the spontaneous polarization normal to the spiral propagation vector $[7,8]$.

Early work on manipulating magnetization by an electric field was reported by Ascher et al., who observed that a reversal of the electric polarization $\mathbf{P}$ of $\mathrm{Ni}_{3} \mathrm{~B}_{7} \mathrm{O}_{13} \mathrm{I}$ from the [001] crystallographic axis to [001] by an electric field leads to a rotation of the weak ferromagnetic moment $\mathbf{m}$ from [110] to [110] [9]. Much later, it was demonstrated that an electric field controls the magnetic order of the $\mathrm{Ho}^{3+}$-ions in hexagonal $\mathrm{HoMnO}_{3}$ and that antiferromagnetic domain patterns correlate with ferroelectric domains in thin $\mathrm{BiFeO}_{3}$ films $[10,11]$. Recently, an elegant way to control the spin helicity by an electric field was discovered in the magnetic spiral compound $\mathrm{TbMnO}_{3}$ [12]: the reversal of spin helicity was achieved by the electric field cooling through $T_{C}$. Nevertheless, the coercive electric field was too large $(>20 \mathrm{kV} / \mathrm{cm})$ to reverse the ferroelectric polarization below the ferroelectric Curie temperature. Despite these few studies, little is known about how magnetic structures are influenced by electric fields.
PACS numbers: 75.80.+q, 61.05.C,- 75.25.+z, 77.80.-e

In the $R \mathrm{Mn}_{2} \mathrm{O}_{5}$ series with $R=\mathrm{Ho}, \mathrm{Tb}, \mathrm{Dy}, \mathrm{Y}$, and $\mathrm{Er}$ the magnetoelectric couplings are gigantic, and the magnetic phases involved are complicated and commonly incommensurate with lattice [5,13-15]. The spontaneous electric polarization $\mathbf{P}$ results from acentric spin-density waves [14] whereas little is known about the underlying mechanism of multiferroicity because of their structural and magnetic complexity. Furthermore, the brake of inversion symmetry in the ferroelectric magnetic phases implies a coupling of magnetism to odd orders of $\mathbf{P}[16,17]$.

$\mathrm{ErMn}_{2} \mathrm{O}_{5}$ shows spontaneous electric polarization along the $b$ axis at $T_{C 1}=39.1 \mathrm{~K}$, i.e., below the Néel temperature of $T_{N 1}=44 \mathrm{~K}[18,19]$. The system enters a commensurate magnetic $(\mathrm{CM})$ phase at $T_{\mathrm{CM}}=37.7 \mathrm{~K}$ with a magnetic ordering vector of $\mathbf{q}=\left(\frac{1}{2}, 0, \frac{1}{4}\right)$ upon cooling from a two-dimensionally-modulated incommensurate magnetic structure (2D-ICM) with $\mathbf{q}=\left(q_{x}, 0, q_{z}\right)$. Thereby the system passes through a one-dimensionallymodulated incommensurate magnetic phase (1D-ICM) with $\mathbf{q}=\left(q_{x}, 0, \frac{1}{4}\right)$. The transition temperature $T_{D}$ between the two incommensurate magnetic phases is correlated to the Curie temperature of the spontaneous electric polarization at $T_{C 1}=T_{D}=39.1 \mathrm{~K}$. In the present paper, we demonstrate that ferroelectricity in $\mathrm{ErMn}_{2} \mathrm{O}_{5}$ strongly couples with the commensurate magnetic structure and that the magnetic order can be deliberately modulated, excited and switched by applying a static electric field.

$\mathrm{ErMn}_{2} \mathrm{O}_{5}$ single crystals were grown by spontaneous crystallization [20]. Resonant soft X-ray magnetic diffraction was performed using the RESOXS station at the SIM beam line of the Swiss Light Source at the Paul Scherrer Institut, Switzerland [21]. The incoming linearly polarized light was oriented either parallel $(\pi)$ or perpendicular $(\sigma)$ to the scattering plane. The sample was mounted on a sapphire plate on a continuous-helium-flow cryostat, providing a temperature range of $29 \mathrm{~K} \leq T \leq 300 \mathrm{~K}$. The $b$ axis was aligned parallel to the scattering plane, and the 

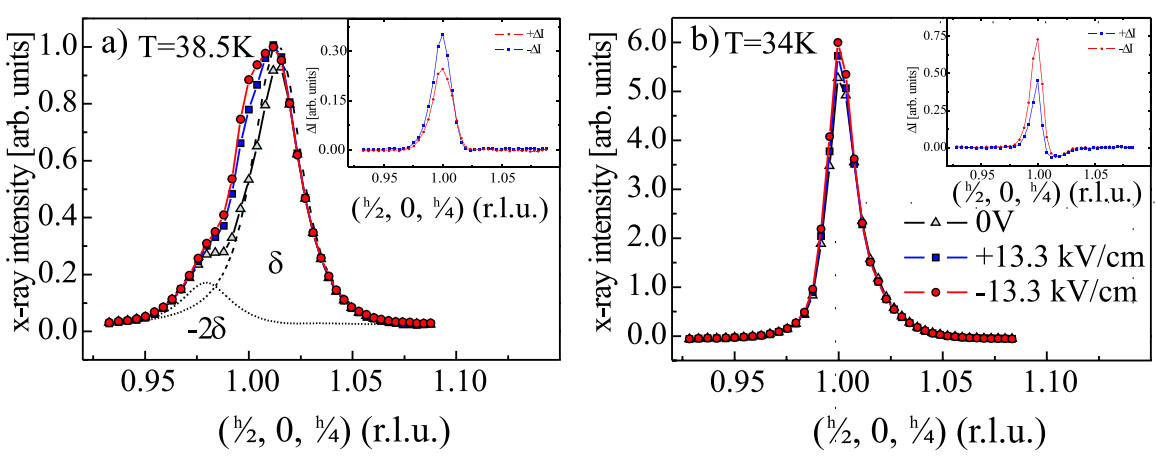

FIG. 1 (color online). $\theta / 2 \theta$ scans of the $\left(\frac{1}{2}, 0, \frac{1}{4}\right)$-reflection of $\operatorname{ErMn}_{2} \mathrm{O}_{5}$ taken at the $\mathrm{Mn} L_{3}$-edge at $T=34 \mathrm{~K}$ below the $2 \mathrm{D}$ to $1 \mathrm{D}-\mathrm{ICM}$ phase transition (a) and above at $T=38.5 \mathrm{~K}$ (b) with $\pi$-polarized incident light. $\delta$ corresponds to the magnetic and $-2 \delta$ to the induced aspheric charge reflection (see text). The inset show the intensity difference $\Delta I^{ \pm}=I( \pm E)-I(0 \mathrm{~V})$. electric field was applied perpendicular to both the $b$ axis and the magnetic wave vector $\left(\frac{1}{2}, 0, \frac{1}{4}\right)$.

In Fig. 1 we present resonant magnetic soft x-ray diffraction of $\mathrm{ErMn}_{2} \mathrm{O}_{5}$ taken with $\pi$-polarized light by $\theta / 2 \theta$ scans at the $\mathrm{Mn} L_{3}$-edge ( $E=643.75 \mathrm{eV}$; probing the $\mathrm{Mn}$ moments only) at two different temperatures, below [Fig. 1(a)] and above the ICM-CM phase transition, respectively. Magnetic Bragg scattering appears below $T_{\mathrm{CM}}$ at $\mathbf{q}=\left(\frac{1}{2}, 0, \frac{1}{4}\right)$, whereas above $T_{\mathrm{CM}}$ two satellite reflections $\delta$ and $-2 \delta$ appear. $\delta$ denotes the deviation in $\mathbf{q}$ from the commensurate values. The resonant magnetic scattering amplitude relevant for the antiferromagnetic reflection in the electric dipole $(E 1)$ approximation can be written as

$$
f_{E 1}^{\mathrm{res}} \propto i\left(\mathbf{e}_{i} \times \mathbf{e}_{f}^{*}\right) \cdot \mathbf{m} F^{(1)}+\left(\mathbf{e}_{i} \cdot \mathbf{m}\right)\left(\mathbf{e}_{f}^{*} \cdot \mathbf{m}\right) F^{(2)},
$$

were $\mathbf{m}$ denotes the local moment direction [22,23]. The first term depends linearly on $\mathbf{m}$ and gives first-harmonic satellites $(\delta)$, whereas the second term corresponds to orbital scattering leading to the second-harmonic satellite $-2 \delta$ (quadratic in $\mathbf{m}$ ), and describes the induced charge anisotropy of Mn which is supported by different polarization and energy dependencies of these reflections.

Applying an electric field of $E= \pm 13.3 \mathrm{kV} / \mathrm{cm}$ perpendicular to the directions of the ferroelectric polarization and the magnetic wave vector $\mathbf{q}$ leads to a pronounced increase of the scattered magnetic intensity at $\mathbf{q}=\left(\frac{1}{2}, 0, \frac{1}{4}\right)$ in the commensurable as well in the 1D-ICM phase as shown in Figs. 1(a) and 1(b). We observe a distinct different magnitude for positive and negative electric field direction $(10 \%)$ which is in relation to the recent findings of the tendency of electric polarization $\mathbf{P}$ to be spontaneously oriented in a preferred direction [19]. Therefore, we conclude this to be a general feature in $\mathrm{ErMn}_{2} \mathrm{O}_{5}$ and not caused by extrinsic and sample dependent effects. The intensity difference $\Delta I^{ \pm}=I( \pm E)-I(0)$ for zero field cooling (ZFC) and for different field cooled (FC) scenarios confirm this findings (data not shown). The insets in Fig. 1 show the intensity difference $\Delta I^{ \pm}$for ZFC. The small dip in the difference intensities on the right side is likely due to a reduction of the intensity from the incommensurate $\delta$ magnetic peak, indicating that the observed difference in the intensity is in part due to a change from 1D-ICM to the $\mathrm{CM}$ phase. Together with the asymmetric peak shape of the commensurate reflection, this is an indication for a coexisting of phases as observed for $\mathrm{YMn}_{2} \mathrm{O}_{5}$ and $\mathrm{TbMn}_{2} \mathrm{O}_{5}$ $[24,25]$. These findings show the direct evidence of manipulation and excitation of the magnetic structure with an in situ applied electric field. To obtain further insight, a detailed temperature dependence of the reflection with and without an electric field was collected, providing an in situ measurement of the coupled magnetic and ferroelectric transitions in $\mathrm{ErMn}_{2} \mathrm{O}_{5}$. The upper part of Fig. 2 presents $\theta / 2 \theta$-scans across the magnetic reflection without any applied electric field in the temperature interval $34 \mathrm{~K} \leq$ $T \leq 44.7 \mathrm{~K}$. The Néel-temperature at $T_{N}=44 \mathrm{~K}$ is observed with the onset of magnetic scattering. The fact that $T_{N}$ is identical for both the $\delta$ and $-2 \delta$ satellites establishes that they originate from magnetic ordering. Obviously, the magnetic spiral (represented by $\delta$ ) drives the aspheric charge density wave $(-2 \delta)$. Fits were used to establish the peak positions of $\delta$ and $-2 \delta$ given by the black dots in

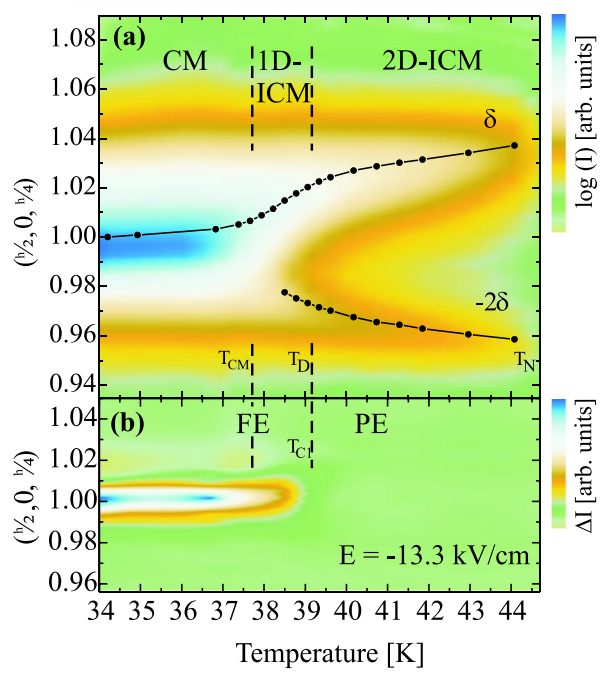

FIG. 2 (color online). (a) Temperature dependence of the magnetic $\left(\frac{1}{2}, 0, \frac{1}{4}\right)$-reflection and the incommensurate $\delta$ and $-2 \delta$ reflections of $\mathrm{ErMn}_{2} \mathrm{O}_{5} . T_{D}=T_{C 1}=39.1 \mathrm{~K}$ is defined by the change in slope of $\delta(T)$ and $-2 \delta(T)$. At $T_{\mathrm{CM}}=$ $37.7 \mathrm{~K}, \mathrm{ErMn}_{2} \mathrm{O}_{5}$ enters the CM. (b) Intensity difference $\Delta I(T)$ as function of temperature. The appearance of $\Delta I$ at $T_{D}$ is explicitly shown in Fig. 3(c). 
Fig. 2(a). By further lowering the temperature to $T_{D}=$ $39.1 \mathrm{~K}$, the positions of $\delta$ and $-2 \delta$ slightly changes. With decreasing temperature, the magnetic structure becomes one-dimensionally-modulated incommensurate (1D-ICM) at $T_{D}=39.1 \mathrm{~K}[18,19]$. The lock-in of $q_{z}$ to the incommensurate value leads to a significant change in the slope of $\delta(T)$ and $-2 \delta(T)$, solely $q_{x}$ changes further on. The phase transition is marked by the first vertical line in Fig. 2. With a further decrease in temperature, $\mathrm{ErMn}_{2} \mathrm{O}_{5}$ enters the commensurate magnetic phase $(\mathrm{CM})$ at $T_{\mathrm{CM}}=37.7 \mathrm{~K}$. At this point $\delta(T)$ is indistinguishable from $-2 \delta(T)$ and both reflections merge into the commensurate $\left(\frac{1}{2}, 0, \frac{1}{4}\right)$ reflection.

Simultaneously, we measure the influence of the applied electric field on the magnetic scattering at each temperature. The lower part of Fig. 2 presents the intensity difference $\Delta I(T)$ between a scan with and without an applied electric field of $E=-13.3 \mathrm{kV} / \mathrm{cm}$. Obviously, the onset of $\Delta I(T)$ is associated with the 2D-ICM to 1D-ICM transition. Since the $E$ field is applied in situ, these results represent direct proof of a coupling between ferroelectric and magnetic order, since no change in the magnetic signal is observed in the paraelectric phase. Moreover, the intensity difference peak appears at the commensurate peak position and $\Delta I$ is stable in $\mathbf{q}$.

Below $T_{C 1}$, Mn spins are excited into the noncollinear commensurate magnetic structure, with the propagation vector $\mathbf{q}=\left(\frac{1}{2}, 0, \frac{1}{4}\right)$ by the application of an electric field. These findings demonstrate the establishment of commensurality in the 1D-ICM phase by the presence of an electric field and hence an influence of the magnetic moments by $E$. More information on the magnetic transitions is available from the integrated intensities $I(T)$ for $\delta$ and $-2 \delta$, which are plotted as a function of temperature in Fig. 3(a). The onset of $I(T)$ coincides with the Néel temperature $\left(T_{N}\right)$ for both satellite reflections. The change in $\mathbf{q}$ for $\delta(T)$ and $-2 \delta(T)$, as observed in Fig. 2(a), is accompanied by a significant change in intensity when passing through the 2D-ICM to 1D-ICM (PE to FE) transition at $T_{D}\left(T_{C 1}\right)$. Finally, the appearance of the commensurate magnetic phase at $T_{\mathrm{CM}}$ is signaled by the merging of $\delta(T)$ and $-2 \delta(T)$ to the commensurate peak, though the results of Fig. 1 indicate that a weak ICM contribution still coexists. In Fig. 3(b), the fit of $I(T)$ at lower temperatures to a power law is given. Extending the fit, the curve crosses zero at approximately $T=37.6 \mathrm{~K}$, indicating the onset of the commensurate magnetic structure. Note that in the case of coexisting phases, as suggested in the discussion of Fig. 1, the fit does not reflect a critical exponent. Figure 3(c) shows $\Delta I(T)$ as function of temperature normalized to the initial intensity. $\Delta I(T)$ follows closely the electric polarization $P_{b}$ measured by Higashiyama et al. with the onset of $\Delta I(T)$ at approximately $T_{C 1} . P_{b}(T)$ is reproduced from Ref. [26], with an adjustment to temperatures measured by Kobayashi et al. and Fukunaga et al.
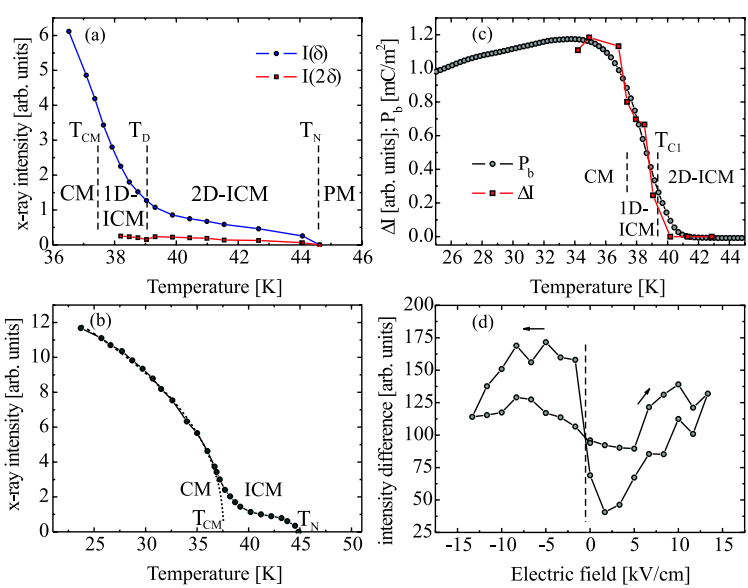

FIG. 3 (color online). (a) X-ray intensity $I$ as function of temperature for the $\delta$ and $-2 \delta$ satellites. (b) The temperature dependence of $I$ (solid line and dots) for $\delta$ follows a power law at low temperatures (dotted line). (c) $\Delta I(T)$ follows the polarization $P_{b}$ for $H=0 \mathrm{~T}$ (reproduced from Ref. [26]). (d) X-ray intensity $I$ as function of the applied electric field at $T=38.5 \mathrm{~K}$, showing its hysteretic behavior after $\mathrm{FC}(+)$. The dotted line shows the bias field.

$[18,19]$. The dependence of the intensity $\Delta I(E)$ as function of the applied electric field is shown in Fig. 3(d). The data were taken after $\mathrm{FC}(+)$ scenario which leads to positive $\Delta I$ values at $0 \mathrm{~V}$ and a negative field bias. As reference we use the $I(0 \mathrm{~V})$ measured with ZFC on exact the same temperature. This allows to obtain a hysteresis by sweeping the electric field $E$. We observe an increasing of $\Delta I$ when increasing $E$ up to $\sim 9 \mathrm{kV} / \mathrm{cm}$, followed by a decreasing of the intensity difference by further increasing the applied electric field up to the maximum value of $E=$ $13.3 \mathrm{kV} / \mathrm{cm}$. Similar behavior could also be observed by applying the electric field in the opposite direction as displayed in the left part of the hysteresis. A second remarkable finding is the difference in the slope of the hysteresis for increasing and decreasing $E$ when passing the $0 \mathrm{~V}$ position after turning the field. It shows that the system is in a different magnetic state as the response to the $E$ field depends significantly on history, despite the fact that the small negative bias already proves the imprint of a magnetic response. The decrease of $\Delta I$ for $E>$ $\pm 9 \mathrm{kV} / \mathrm{cm}$ is likely the origin of the appearance of the hysteresis and causes the switching of the magnetic states. One would therefore expect, that smaller $E$ fields, though still leading to a change of intensity, show no hysteresis. Earlier studies seems to support this assumption as they reveal that $\mathrm{ErMn}_{2} \mathrm{O}_{5}$ shows an unusual magnetic field dependence of magnetoelectric polarization leading to complicated $P-H$ hysteresis, whereas only high order terms up to the fourth order could describe the results satisfactory [27,28]. Our measurements of the hysteretic behavior of the magnetic Bragg intensity show a clear memory effect of $\Delta I(E)$. Moreover, the slope of the hys- 
teresis depends significantly on history. Nevertheless, this measurement is a clear indication that the magnetism in $\mathrm{ErMn}_{2} \mathrm{O}_{5}$ can be switched between two different states by the electric field. Additionally, a difference in $\Delta I$ of approximately $15.3 \%$ is observed between the $\mathrm{FC}(+)$ and FC $(-)$ field cooled scenarios.

Finally, we probe the polarization dependency of the intensity difference $\Delta I$. For $0 \mathrm{~V}$, we find the intensity ratio between $\sigma$ and $\pi$ polarized light to be $\pi /\left.\sigma\right|_{0 \mathrm{v}}=2.16$. Applying an electric field leads to ratios of $\pi /\left.\sigma\right|_{-\Delta E}=$ 2.08 and $\pi /\left.\sigma\right|_{+\Delta E}=2$.91. Since $\sigma \sigma$-scattering is absent for magnetic scattering and assuming the contribution of the orbital scattering to be small, the change in the intensity ratios is an indication that the direction of the magnetic moments is changed by applying an electric field rather than a simple enhancement of the moments. Based on the orthogonal character of the DM interaction, one would assume that an electric field applied perpendicular to both, the direction of the ferroelectric polarization and $\mathbf{q}$, would induce a magnetic moment along the crystallographic $b$ axis. Since the manganese magnetic moments have components along all three crystallographic directions [14] and considering that the magnetic structure is ambiguous, the influence on the structure factor cannot be determined quantitatively. However, this significant change observed in the polarization ratio indicates that the electric field rotates the magnetic moments leading to an electric field dependent commensurate magnetic structure.

In summary, resonant magnetic soft x-ray diffraction experiments were performed on multiferroic $\mathrm{ErMn}_{2} \mathrm{O}_{5}$. Applying a static electric field leads to a significant increase of the magnetic scattering intensity. The difference in scattered intensity clearly demonstrates the generation of magnetic scattering intensity at the commensurate $\left(\frac{1}{2}, 0\right.$, $\frac{1}{4}$ ) position which is stable in q. The appearance of intensity difference $\Delta I(T)$ as function of temperature reveals the coincidence of the 2D-ICM to 1D-ICM magnetic transition and the para- to ferroelectric transition. In the ferroelectric phase, an applied electric field pushes the system into the commensurate magnetic phase by changing the direction of the magnetic moments. Hysteresis loops as well as ZFC and $\mathrm{FC}$ experiments reveal the possibility to imprint a magnetic response function by an electric field.

We have benefited from valuable discussions with $\mathrm{S}$. Lovesey, S. Gvasaliya, and B. Roessli and from the experimental support of the X11MA beam line staff. The work was partially supported by the by RFBR grants Nos. 05-0217822 and 05-02-16328 and by Presidium of Russian Academy of Sciences Grant No. P3. The financial support of the Swiss National Science Foundation is grateful acknowledged.

[1] M. Fiebig, J. Phys. D 38, R123 (2005).
[2] Y. Tokura, Science 312, 1481 (2006).

[3] S.-W. Cheong and M. Mostovoy, Nat. Mater. 6, 13 (2007).

[4] T. Kimura, T. Goto, H. Shintani, K. Ishizaka, T. Arima, and Y. Tokura, Nature (London) 426, 55 (2003).

[5] N. Hur, S. Park, P. A. Sharma, J.S. Ahn, S. Guha, and S.-W. Cheong, Nature (London) 429, 392 (2004).

[6] P. G. Radaelli and L. C. Chapon, Phys. Rev. B 76, 054428 (2007).

[7] I. A. Sergienko and E. Dagotto, Phys. Rev. B 73, 094434 (2006).

[8] H. Katsura, N. Nagaosa, and A. V. Balatsky, Phys. Rev. Lett. 95, 057205 (2005).

[9] E. Ascher, H. Rieder, H. Schmidt, and H. Stssel, J. Appl. Phys. 37, 1404 (1966).

[10] T. Lottermoser, T. Lonkai, U. Amann, D. Hohlwein, J. Ihringer, and M. Fiebig, Nature (London) 430, 541 (2004).

[11] T. Zhao, A. Scholl, F. Zavaliche, K. Klee, M. Barry, A. Doran, M. P. Cruz, Y.H. Chu, C. Ederer, and N. A. Spaldin et al., Nat. Mater. 5, 823 (2006).

[12] Y. Yamasaki, H. Sagayama, T. Goto, M. Matsuura, K. Hirota, T. Arima, and Y. Tokura, Phys. Rev. Lett. 98, 147204 (2007).

[13] L. C. Chapon, G. R. Blake, M. J. Gutmann, S. Park, N. Hur, P. G. Radaelli, and S.-W. Cheong, Phys. Rev. Lett. 93, 177402 (2004).

[14] L. C. Chapon, P. G. Radaelli, G. R. Blake, S. Park, and S.-W. Cheong, Phys. Rev. Lett. 96, 097601 (2006).

[15] C. R. dela Cruz, F. Yen, B. Lorenz, M. M. Gospodinov, C. W. Chu, W. Ratcli, J. W. Lynn, S. Park, and S. W. Cheong, Phys. Rev. B 73, 100406(R) (2006).

[16] M. Kenzelmann, A. B. Harris, S. Jonas, C. Broholm, J. Schefer, S. B. Kim, C.L. Zhang, S.-W. Cheong, O. P. Vajk, and J. W. Lynn, Phys. Rev. Lett. 95, 087206 (2005).

[17] T. Arima, A. Tokunaga, T. Goto, H. Kimura, Y. Noda, and Y. Tokura, Phys. Rev. Lett. 96, 097202 (2006).

[18] S. Kobayashi, T. Osawa, H. Kimura, Y. Noda, I. Kagomiya, and K. Kohn, J. Phys. Soc. Jpn. 73, 1031 (2004).

[19] M. Fukunaga, K. Nishihata, H. Kimura, Y. Noda, and K. Kohn, J. Phys. Soc. Jpn. 76, 074710 (2007).

[20] V. A. Sanina, L. M. Sapozhnikova, E. I. Golovenchits, and N. V. Morozov, Sov. Phys. Solid State 30, 1736 (1988).

[21] U. Staub, V. Scagnoli, A. M. Mulders, K. Katsumata, Z. Honda, H. Grimmer, M. Horisberger, and J.M. Tonnerre, Phys. Rev. B 71, 214421 (2005).

[22] J. P. Hannon, G. T. Trammell, M. Blume, and D. Gibbs, Phys. Rev. Lett. 61, 1245 (1988).

[23] S. W. Lovesey, E. Balcar, K.S. Knight, and J.F. Rodriguez, Phys. Rep. 411, 233 (2005).

[24] S. Kobayashi, T. Osawa, H. Kimura, Y. Noda, I. Kagomiya, and K. Kohn, J. Phys. Soc. Jpn. 73, 1593 (2004).

[25] J. Okamoto, D. J. Huang, C.-Y. Mou, K. S. Chao, H.-J. Lin, S. Park, S.-W. Cheong, and C. T. Chen, Phys. Rev. Lett. 98, 157202 (2007).

[26] D. Higashiyama, S. Miyasaka, and Y. Tokura, Phys. Rev. B 72, 064421 (2005).

[27] H. Nakamura and K. Kohn, Ferroelectrics 204, 107 (1997).

[28] Y. Koyata and K. Kohn, Ferroelectrics 204, 115 (1997). 